\title{
Object-based attention: Shifting or uncertainty?
}

\author{
Leslie Drummond and Sarah Shomstein \\ George Washington University, Washington, D.C.
}

\begin{abstract}
The contribution of object-based attentional guidance to visual processing is widely accepted, and recent models of attentional selection now include both space- and object-based representations. Nevertheless, although the mechanism of space-based attentional selection has been well characterized, the mechanism underlying object-based attentional selection remains poorly understood. Recent attempts at identifying the mechanism giving rise to object-based attentional selection have put forth two conflicting alternatives. The first suggests that object-based effects are driven by attentional uncertainty (i.e., the attentional prioritization hypothesis), whereas the second suggests that objects guide attentional selection only when an attentional shift is necessitated (i.e., the attentional-shifting hypothesis). Here, we directly investigated which of the two suggested accounts drives object-based effects, by pitting attentional certainty against attentional shifting. In a series of four experiments, we manipulated the certainty of target location, shifting of attention, and the stimulus onset asynchrony. It was observed that object-based effects depended solely on certainty of the forthcoming target location. These results suggest that attentional prioritization, and not a mere shift of attention, gives rise to object-based guidance of attentional selection. In addition, these results lend further support to the attentional prioritization account of object-based attention and provide further constraints on the mechanisms of object-based selection.
\end{abstract}

Early studies of object-based attentional selection focused almost entirely on demonstrating that object-based representations contribute to attentional guidance (Brawn \& Snowden, 2000; Duncan, 1984; Rock \& Guttman, 1981) and that object-based contributions to selection coexist with space-based orienting (Egly, Driver, \& Rafal, 1994; Goldsmith, 1998; Moore, Yantis, \& Vaughan, 1998; Müller \& Kleinschmidt, 2003). A large body of evidence in support of object-based attentional selection has been a product of a two-rectangle paradigm, originally developed by Egly et al. In this paradigm, subjects are presented with two rectangles oriented either horizontally or vertically. The geometry of this display is such that the rectangles, as a group, form a perfect square with each end positioned equidistantly from the opposite end. After a brief delay, one end of one of the rectangles is cued by a local thickening of the object outline. Following the cue, a target is presented at the location previously occupied by the cue (validly cued location), at the location opposite the cue within the cued rectangle (same-object invalid location), or at the equidistant location in the uncued rectangle (different-object invalid location).

Two major findings have emerged from this paradigm: (1) Targets that appear in the same spatial location as the cue (i.e., valid cue) are detected more quickly than those that appear elsewhere (i.e., invalid cue), a finding taken as evidence for space-based attentional orienting; and (2) targets appearing the same distance away from the cue at the same-object location are detected more quickly than those appearing at the different-object location, a finding taken as evidence for object-based attentional orienting. The latter result cannot be explained by spatial selection alone, since the distance between the cued location and both invalid locations is exactly the same; nor is it merely an effect of crossing the boundaries of the rectangles, since the effect has been demonstrated with occlusion and under various grouping scenarios (Behrmann, Zemel, \& Mozer, 1998; Moore et al., 1998; Shomstein \& Behrmann, 2008; Watson \& Kramer, 1999; Zemel, Behrmann, Mozer, \& Bavelier, 2002). This paradigm has been an instrumental tool in demonstrating the contributions of space-based and object-based representations to attentional guidance.

Recent studies, however, have shifted their focus away from demonstrating the coexistence of space- and objectbased attentional guidance, to elucidating mechanisms that give rise to object-based attentional guidance (Avrahami, 1999; Cepeda \& Kramer, 1999; Chen \& Cave, 2006, 2008; Lamy \& Egeth, 2002; Shomstein \& Behrmann, 2006,

L. Drummond, drummond@gwmail.gwu.edu 
2008; Shomstein \& Yantis, 2002, 2004). Currently, there are three general accounts that are proposed as possible mechanisms subserving object-based attentional orienting. The first hypothesis maintains that object-based attention arises from the spread of attention (object-guided spatial selection) and is termed sensory enhancement. According to this account, when spatial attention is directed to one part of an object, what follows is facilitation of sensory processing for all spatial locations within that object. Attention spreads automatically from the cue to encompass other regions within the object's boundaries-hence, the enhancement of all features and parts of the single object (Chen \& Cave, 2006, 2008; Richard, Lee, \& Vecera, 2008; Roelfsema, Lamme, \& Spekreijse, 1998). It follows from this account that attending to a part of an object will involuntarily and mandatorily result in the "spread of attention" throughout an entire object (Corbetta, Miezin, Dobmeyer, Shulman, \& Petersen, 1990; O’Craven, Downing, \& Kanwisher, 1999).

Two alternative hypotheses to the sensory enhancement account have recently emerged: one proposed by Lamy and Egeth (2002) and labeled here as the attentionalshifting hypothesis, and the other proposed by Shomstein and Yantis $(2002,2004)$ and labeled here as the attentional prioritization hypothesis. We consider each one in turn.

Lamy and Egeth (2002) sought to investigate the mechanism of object-based attentional guidance by examining specific circumstances that tend to give rise to objectbased effects. It was proposed that a critical factor in determining whether objects will guide attentional selection is whether the perceptual task at hand requires shifts of spatial attention. Rather than attention necessarily spreading through a cued object, object-based effects are predicted only when attentional shifts are executed. In a set of four experiments, the authors first replicated the standard object-based effect, using a variant of the Egly et al. (1994) two-rectangle paradigm that replaced target detection with a same/different judgment task. In this version of the paradigm, the presentation of two rectangles was followed by the appearance of two target shapes (squares) that either matched or differed in size (i.e., large or small). The first manipulation involved removing the cue and presenting target shapes simultaneously, $1,000 \mathrm{msec}$ after the presentation of two rectangles. Under such conditions, the authors failed to observe object based effects, such that reaction time (RT) to judge the two squares as same or different did not depend on whether they appeared within the same or different rectangles (for a similar result, see Watson \& Kramer, 1999). In a subsequent experiment, the authors slightly altered the paradigm by presenting the two targets in rapid succession, rather than simultaneously, while again asking subjects to perform the sizematching task. With targets presented in quick succession, object-based effects were observed, such that RTs were shorter for matching judgments when both squares appeared within the same object than when the two squares appeared in different objects. Lamy and Egeth interpreted the observed findings within the context of attentional shifting, concluding that the reason for observing objectbased effects in a successive target presentation paradigm, and not in the simultaneous presentation paradigm, was the fact that successive target matching required subjects to shift their attention from one target to the other, whereas the simultaneous target presentation task did not require attentional shifts. It was concluded that object-based effects are elicited solely by a shifting mechanism. In other words, attentional shifts are faster (and more precise) when executed within a single object than when the same spatial shift requires spanning multiple objects. Note that according to the sensory enhancement account, object-based effects would have been present in all of the manipulations varied by Lamy and Egeth. However, object-based effects were present only when attentional shifts were necessitated, and as such, these results offered strong evidence in support of the attentional-shifting hypothesis and against the sensory enhancement account.

Shomstein and Yantis (2002) offered an alternative explanation that challenges both the sensory enhancement and the attentional-shifting hypotheses. In what has been termed the attentional prioritization hypothesis, the authors argued that object-based effects can be attributed to a simple attentional prioritization mechanism. Following complex inputs to the visual system, and all things being equal (i.e., uncertainty regarding the location of the future target), the organism learns to assign higher attentional priority to locations within an already attended object than to locations elsewhere (resulting in object-based effects). Given that attentional priority determines the order in which the visual scene will be investigated, unattended parts of an attended object will enjoy an attentional advantage (enhancement) over other objects and locations in a scene, thus giving rise to object-based effects. It is useful to view this account in the context of Wolfe's (1994) Guided Search, suggesting that the order of search is governed by attentional priorities that are affected not only by local feature contrast and by similarity to a target template (Cave \& Wolfe, 1990; Wolfe, 1994), but also by object structure. It should be noted that although here, and elsewhere (Shomstein \& Yantis, 2002), we discuss attentional prioritization as an ordering of the search, a strictly serial search need not be warranted, and an equally plausible implementation would be display-wide parallel processing with different rates of information extraction according to attentional priority.

The term enhancement in attentional prioritization and sensory enhancement theories refers to an increased efficiency in processing an object, a spatial location, or a part of an object. In this way, defining sensory enhancement as a mechanism is a misnomer; perhaps the more appropriate description would be attentional spreading. The dividing factor is the mechanism via which the objects or location representations are enhanced. Sensory enhancement necessitates an automatic spread of attention along object boundaries. Attentional prioritization, on the other hand, explains the enhancement by way of priority assignments, such that the same object location is enhanced by way of the cue, but only when there is no alternative strategy in place to determine the most likely target location (e.g., "target always appears one location clockwise"). When the target location is certain, the highest priority is assigned to 
that single location, and enhancement takes place at only that location. Prioritization does assume that sensory representations of the display (or the target location, in terms of certainty) are enhanced. However, the sensory enhancement theory presumes that the process of enhancement is automatic from the cued location to all other same-object locations, whereas prioritization suggests an intentional, voluntary (although not explicit) application of enhancement to the priority map of the display, on the basis of knowledge given about the contingencies of the scene.

In most variants of the two-rectangle paradigm, both the sensory enhancement and the attentional prioritization hypotheses lead to similar results - namely, the emergence of an object-based effect. However, the two accounts make different predictions under some circumstances. One such circumstance was investigated by Shomstein and Yantis (2002), where subjects were asked to identify a central target while attempting to ignore flanking distractors that were mapped to either a compatible or an incompatible response (following the classic flanker paradigm of Eriksen and colleagues; see, e.g., Eriksen \& Eriksen, 1974). The flanking letters appeared either within the object that the target occupied or at an equidistant location in a different object. An important element of this design was that the spatial location of the target was known with $100 \%$ certainty. According to the sensory enhancement account, attention "spreads" within the attended object, so that the flanking letters located in that object should benefit perceptually, thus causing more interference, as compared with objects elsewhere. This account predicts, therefore, that the time to identify the target letter will depend on the identity (or more precisely, on the response compatibility) of the flanking letters to a greater degree when they appear in the attended object than when they appear in the unattended object. In contrast, the attentional prioritization account predicts that because the target location is known with $100 \%$ certainty, only one location should be examined in order to perform the task, so the priority of other locations in the scene should remain near zero. This account consequently predicts that flanker interference will not depend on whether the flankers occupy the same or a different object as the target (although the absolute spatial separation between the target and flankers may well affect performance, due to spatial "leakage" of attention). The predictions of the prioritization account, and not of the sensory enhancement, were satisfied (see also Shomstein \& Behrmann, 2008; Shomstein \& Yantis, 2004); that is, there was no modulation of flanker effect size by whether flankers appeared on the same or a different object. To summarize, sensory enhancement suggests that attention simply spreads from the cued location within an object, whereas prioritization describes this process as being purposely strategic (i.e., attentional priority is actively assigned on the basis of knowledge about the relationship between the cue and target locations, such that an object is enhanced only if no alternative strategy is available).

Although both the attentional-shifting and the attentional prioritization accounts challenged the enhancement mechanism, ${ }^{1}$ neither could dismiss the validity of the other. In prior investigations, attentional shifting and target location certainty were confounded. In other words, either the paradigm required shifting while target location was uncertain, or target locations were certain but shifts were unnecessary. In Lamy and Egeth (2002), for example, subjects were asked to shift their attention between two successively presented targets that appeared in random locations within the display; in other words, attentional shifts were required to perform the task, and target locations were unknown. In Shomstein and Yantis's (2002) flanker paradigm, on the other hand, subjects were not required to shift their attention, and the target location was known in advance (i.e., the target was always presented in the center). In order to directly compare these two alternative accounts of object-based attentional guidance and to determine which one is responsible for driving the object-based effect, attentional shifting and attentional certainty have to be juxtaposed.

The purpose of the present study, then, was to dissociate attentional shifts from target position certainty, in order to examine which of the two mechanisms, attentional shifting or attentional prioritization, gives rise to object-based attentional guidance.

\section{EXPERIMENT 1}

Experiment 1 provides an initial investigation into whether attentional shifts or attentional certainty give rise to object-based guidance of attentional selection. This experiment adopted the two-rectangle cuing paradigm of Egly et al. (1994), while dissociating attentional shifting from attentional certainty. Attentional certainty was induced via a tight correlation between the spatial location of the cue and the location of the upcoming target. Although the target location varied from trial to trial, certainty of the upcoming target location was $100 \%$, because the target location was always tied to the location of the preceding cue; that is, the target appeared one location clockwise from the cue. Since the target location was never at the same position as the cue, subjects had to execute an attentional shift to the target location in order to perform the imposed target discrimination task. Therefore, in this manipulation, subjects executed attentional shifts while having full certainty as to the location of the upcoming target. In addition, the stimulus onset asynchrony (SOA) was manipulated in order to determine the time course of object-based and prioritization effects. Since the position of the target was directly linked to the cued location, we hypothesized that a finite amount of time had to pass in order for the subject to interpret the location of the cue and to then apply the target position certainty rule (e.g., applying the knowledge that if the top right location was cued, the target would appear in the bottom right location).

It is important to note that this experiment successfully disentangles attentional shifting from attentional certainty and is designed in such a way as to ensure that both hypotheses make different predictions. According to the attentional-shifting hypothesis, object-based effects should emerge at all SOAs simply as a result of the shift of attention, whereas the attentional prioritization hypothesis predicts different results across time. Namely, object- 
based effects should modulate target processing at early SOAs (i.e., with insufficient time to create the priority map) and should not modulate target processing at longer SOAs, because once a location is prioritized, attention is focused on the target location and the object-based representation is no longer needed to guide attention.

\section{Method}

Subjects. Forty-six undergraduate students (22 male) at George Washington University participated for extra credit in psychology courses. Fourteen subjects took part in the SOA 200-msec condition, 15 took part in the SOA 400-msec condition, and 17 took part in the SOA $600-\mathrm{msec}$ condition. All reported normal or correctedto-normal visual acuity and were naive as to the purpose of the experiment.

Apparatus and Stimuli. The stimulus set was displayed on a 15 -in. monitor at a resolution of $1,024 \times 768$ pixels, using a Dell Optiplex 745. Subjects were seated approximately $60 \mathrm{~cm}$ away from the monitor. The two-rectangle display, targets, and distractors were rendered in white and were presented on a black background. A $0.4^{\circ} \times 0.4^{\circ}$ white fixation cross was presented between the two rectangles, which subtended $4.2^{\circ} \times 4.2^{\circ}$ of visual angle with a $1.8^{\circ}$ rectangle-to-rectangle separation between.

The cue was a red line $\left(0.2^{\circ}\right.$ thick $)$ that abutted the white outline at one end of one of the rectangles. One target ( $\mathrm{T}$ or $\mathrm{L}$ ) and three distractors $(\mathrm{T} / \mathrm{L}$ hybrid created by superimposing the two target letters) appeared on each trial, each subtending $0.47^{\circ} \times 0.33^{\circ}$ of visual angle. The targets and distractors were centered in the ends of the rectangles and were presented at one of four possible orientations: upright $\left(0^{\circ}\right), 90^{\circ}, 180^{\circ}$, or $270^{\circ}$ (see Figure 1).

Design and Procedure. A 2 (object type: same object, different object) $\times 3$ (SOA: 200,400 , or $600 \mathrm{msec}$ ) factorial design was used. Object type was a within-subjects factor, whereas SOA was a between-subjects factor. Display orientation order (horizontal or vertical rectangles first) was counterbalanced across subjects, which was designed to eliminate order effects in the data. The location of the cue was determined randomly on each trial, whereas the target letter was positioned one location clockwise from the cued position. Distractors were assigned to the other three nontarget locations and were selected with replacement.

Subjects completed a total of 896 trials, with 14 blocks of 32 trials for each of the two display orientations. Self-timed breaks were inserted between each block. Subjects were explicitly informed that the target letter would always appear one location clockwise from the cued location (e.g., if the cue appeared in the upper left location, the target would appear in the upper right location). One half of the trials in each block were same-object (SO) trials (target appeared in the same rectangle as the cue), and the other half were differentobject (DO) trials (target appeared in the noncued rectangle), with each trial appearing in random order. Whether the target was an $\mathrm{SO}$ trial or a DO trial depended on the cued location and the orientation of the rectangles (e.g., in the vertically oriented rectangles, a bottom left location cue resulted in a target appearing one location clockwise away from the cue in the top left location, making this a same-object target). What is important to note is that this version of the two-rectangle paradigm does not include trials on which targets appear within the cued location (valid trials).

Each trial began with a display that consisted of the two rectangles and a fixation cross (see Figure 1). After 1,000 msec, the red cue appeared, indicating the location of the upcoming target (one location clockwise). The cue was displayed for $100 \mathrm{msec}$ and was followed by one of the three interstimulus intervals (ISIs). The target display contained a $\mathrm{T}$ or an $\mathrm{L}$ in a corresponding target location (as determined by the cue) and distractors in the nontarget locations. This display was presented until a response was made. The intertrial interval (ITI) was 1,500 msec following each correct response. Each error was followed by visual feedback and an ITI of 3,000 msec.

\section{Results}

Only RTs for correct responses were included in the analysis. RTs were subjected to a trimming procedure (since unlimited target exposure was used), so that those longer than 1,500 msec and shorter than $200 \mathrm{msec}$ were removed, resulting in a total of $3.7 \%$ of the trials being discarded across the three SOAs $(200 \mathrm{msec}, 1 \%$; $400 \mathrm{msec}, 1.2 \%$; $600 \mathrm{msec}, 1.5 \%)$. A one-way repeated measures ANOVA on error rates revealed no significant effects in any of the SOAs $(F<1)$; therefore, only RT data are described below. A preliminary RT analysis revealed that there were neither significant main effects nor interactions involving target identity, rectangle orientation, or cue location (all $F \mathrm{~s}<1$ ); thus, the data were collapsed across these variables. Figure 2 summarizes the findings separately for each SOA.

A one-way repeated measures ANOVA was conducted for each SOA independently. For an SOA of $200 \mathrm{msec}$, an ANOVA revealed a significant main effect of object type $[F(1,14)=37.83, p<.01]$ : SO trials $(M=643 \mathrm{msec})$ were faster than DO trials $(M=658 \mathrm{msec})$, yielding a 15 -msec object-based effect. For an SOA of $400 \mathrm{msec}$, an ANOVA revealed no significant main effect of object type $(F<2.5)$ : SO trials $(M=562 \mathrm{msec})$ were not significantly different from DO trials $(M=568 \mathrm{msec})$. For an SOA of $600 \mathrm{msec}$, an ANOVA revealed no significant main effect of object type $(F<2)$, with SO trials $(M=$

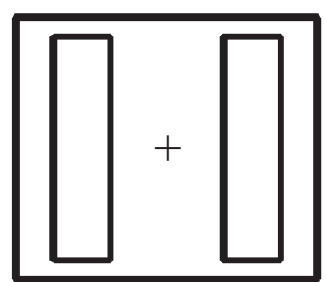

Fixation $1,000 \mathrm{msec}$

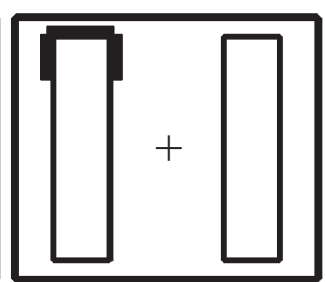

Cue

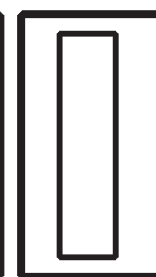

IS $100 \mathrm{msec}$

100,300 , or $500 \mathrm{msec}$

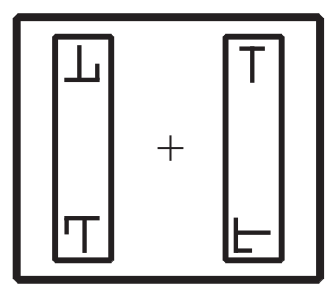

Target

Figure 1. Stimuli and sequence of events in Experiment 1. Fixation was presented for $1,000 \mathrm{msec}$, followed by a red cue for $100 \mathrm{msec}$ and then an interstimulus interval (ISI) of 100, 300, or $500 \mathrm{msec}$. According to the imposed cue-to-target relationship, the target could appear only in the location one position clockwise from the cue. Depending on the location of the cue, the target was presented in either the same or a different object (only the different-object trial is presented here). 


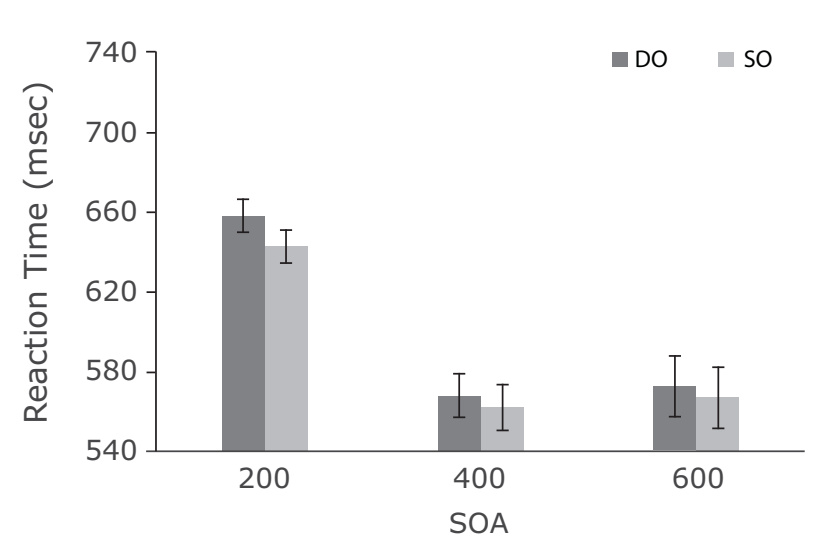

Figure 2. Median correct reaction times for the invalidly cued trials in Experiment 1 for the three stimulus onset asynchrony manipulations (SOA 200, 400, and $600 \mathrm{msec}$ ) for the differentobject (DO) and same-object (SO) target locations.

$551 \mathrm{msec})$ not differing significantly from DO trials $(M=$ $556 \mathrm{msec}$ ) (see Figure 2).

Finally, an ANOVA was conducted to investigate the interaction between SOA and object-based attentional effects across the three SOAs. There was a marginally significant interaction of SOA and same- versus different-object RT $[F(1,47)=2.786, p=.072]$. Planned comparisons between SOAs 200 and 400, SOAs 400 and 600, and SOAs 200 and $600 \mathrm{msec}$ revealed that the size of the object-based effect was clearly modulated by the length of the SOA, such that as SOA increased, the object-based effect size decreased. An ANOVA for 200- versus 400-msec SOAs revealed a significant SOA $\times$ object type interaction $[F(1,28)=6.39$, $p<.02]$ : The object-based effect was significant only for SOA 200 (15 msec), but not for SOA 400 (6 msec). Similarly, an ANOVA on 200- versus 600-msec SOAs revealed a significant SOA $\times$ object type interaction $[F(1,33)=$ 4.184, $p<.05]$ : The object-based effect was significant only for SOA 200 (15 msec) but not at SOA 600 (5 msec). Finally, an ANOVA for 400- versus 600-msec SOAs revealed no significant interaction $(F<1)$.

\section{Discussion}

The results of Experiment 1 demonstrate that objectbased effects are modulated by certainty (i.e., knowing a target's location in advance), rather than by attentional shifts. We hypothesized that if object-based effects are, in fact, driven by attentional prioritization (i.e., certainty of the target's location), object-based effects should be observed at a short SOA (200 msec), because the 100-msec delay between the cue and the target would be insufficient to apply the derivation of the cue-to-target-location relationship, preventing the prioritization map from being established. At longer SOAs, however, since there is more time available to derive the location of the upcoming target based on the location of the cue, target certainty, and not object representations, will guide attention, resulting in the absence of the object-based effect.

In line with our predictions, we observed that at a short SOA (200 msec), a 15-msec object-based effect was pres- ent. This is due to the fact that, even under conditions of certainty, a 100-msec delay between the cue offset and the target onset is not enough time either to derive the relationship between the target and the cue or to effectively prepare for the target at the derived target location (see Shomstein \& Yantis, 2004, for a similar result). In other words, subjects were still in the process of applying the rules of the cue-to-target relationship ("one location clockwise") and were unable to prioritize the location of the upcoming target before the target display appeared. Therefore, at short SOA, objects guide attentional selection, as is evidenced by the presence of a strong object-based effect.

The 400-msec and 600-msec SOA conditions, however, demonstrated the effect of certainty on object-based attention. When the target location was $100 \%$ certain and there was enough time to apply knowledge of the target's location (unlike in the SOA 200 manipulation), the result was no difference between same- and different-object RTs. With a longer SOA, there was enough time afforded for the certainty process to apply the cue-to-target relationship, thereby allowing attention to prioritize the target location. Therefore, given enough time, and in the presence of an alternative strategy (attentional certainty), attentional selection is not guided by object-based representations and is guided by certainty alone. Knowing where the target will appear with $100 \%$ certainty allows attentional selection to prioritize a specific spatial location and to ignore the corresponding object-based representations. It is important to note that according to the attentional-shifting hypothesis, subjects shifted attention from the cued location to the target location for each of the three SOAs, yet only the short SOA manipulation yielded object-based effects, providing a first piece of evidence against the attentional-shifting hypothesis.

\section{EXPERIMENT 2}

In Experiment 1, we demonstrated that target location certainty, and not attentional shifting, predicted whether object representations modulate attentional selection. However, it might be argued that the absence of an objectbased attentional modulation effect in Experiment 1 (SOA 400-msec and SOA 600-msec conditions) can be attributed to a lack of statistical power. For instance, the SOA 400-msec condition still shows a small, although not significant, 6-msec difference between the same-object and different-object trials.

The purpose of Experiment 2, then, was to rule out the possibility that our original paradigm lacks sufficient statistical power to detect significant effects. Therefore, in this experiment, although focusing only on SOA 400, we added a valid cue condition to exploit the fact that targets presented at the validly cued spatial location are detected more efficiently than those positioned at the invalidly cued locations (see Figure 3). This manipulation allowed for contrasting and comparing object-based effects with space-based effects and to ensure that enough power exists to detect the difference between the two types of attentional orienting. If a space-based effect is revealed in the 


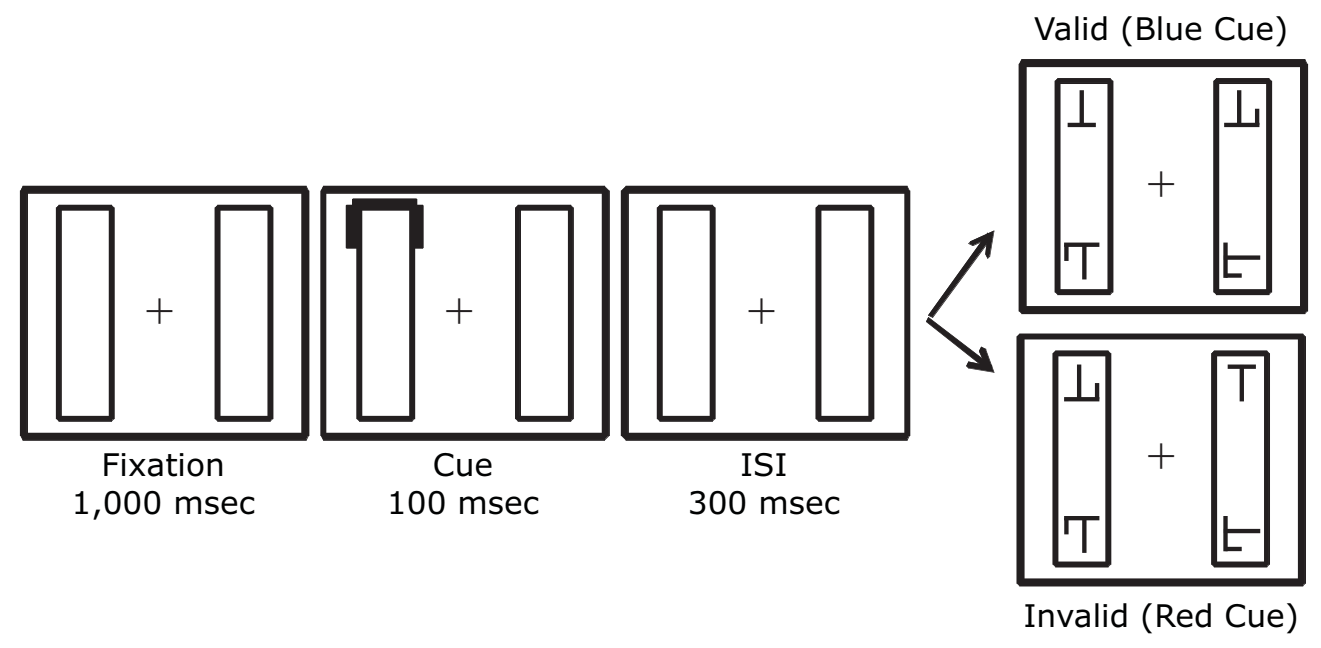

\begin{abstract}
Figure 3. Stimuli and sequence of events in Experiments 2 and 3. In Experiment 2, there were two possible cue-to-target relationships: valid (blue cue), in which the target appeared in the same location as the cue (upper panel), and the invalid (red cue), in which the target appeared one location clockwise from the cue (bottom panel). In Experiment 3, the same valid condition was employed; however, the invalid red cue no longer had any predictive value (target could appear in the location either clockwise or counterclockwise from the cue).
\end{abstract}

absence of the object-based effect, it will be possible to set aside the criticism of insufficient power and will also allow for a replication of the findings observed in Experiment 1 , lending further supporting evidence to the initial finding that attentional prioritization, and not attentional shifting, drives object-based effects.

\section{Method}

Subjects. Eighteen undergraduate students (4 male) from George Washington University participated in return for extra credit. All reported normal or corrected-to-normal visual acuity and were naive as to the purpose of the experiment.

Apparatus and Stimuli. The stimulus set was displayed on a 19 -in. monitor at a resolution of $1,280 \times 1,024$ pixels. All display measurements were the same as those in Experiment 1. In addition to the red cue, a blue cue of the same dimensions was added to indicate that the target would appear in the same location; that is, a validly cued trial. The red cue was termed invalid, and the blue cue valid, in accordance with their predicted relationship with the upcoming target. Subjects were informed that the blue cue indicated that an upcoming target would appear in the same spatial location as the cue, whereas the red cue indicated that an upcoming target would appear one location clockwise away from the cued location. For example, for valid (blue) cues, if the cue appeared in the upper left location, the target always appeared in that same location; for invalid (red) cues, if the cue appeared in the upper left location, the upcoming target appeared in the upper right location. Note that the cue-to-target relationship for the red cue conditions (invalidly cued targets) was exactly the same as that in Experiment 1. All other parameters were kept identical to those in Experiment 1.

Design and Procedure. A 3 (validity: valid, invalid same object, invalid different object) $\times 2$ (display orientation: horizontal, vertical) factorial design was used. Validity was a within-subjects factor, and display orientation was a between-subjects factor. SOA was held constant at $400 \mathrm{msec}$.

Subjects completed a total of 800 trials with either a horizontal or a vertical display. Trials consisted of $60 \%$ validly cued trials and $40 \%$ invalidly cued trials (evenly split between the DO and SO trials). As in Experiment 1, each trial was initiated with the presentation of two rectangles and the fixation cross. After $1,000 \mathrm{msec}$, a blue or red cue was presented at the end of one rectangle for $100 \mathrm{msec}$. If the cue was blue, subjects were informed that the target would appear in the same location. If the cue was red, subjects were informed that the target would appear one location clockwise away from the cue. After a 300-msec ISI, target and distractors were presented and remained on the screen until a response was made. In the case of a correct response, a blank screen (ITI) was presented for 1,500 msec before the next trial began. Incorrect responses resulted in a feedback screen that appeared for an additional 1,500 msec.

\section{Results and Discussion}

Only correct responses were included in the analysis. RTs longer than 1,500 msec and shorter than $200 \mathrm{msec}$ were removed according to the trimming procedure, resulting in fewer than $3 \%$ of the trials being discarded. A one-way repeated measures ANOVA on error rates revealed a significant effect of validity $[F(1,17)=6.541$, $p<.05$ ], in that invalid trials were $1.4 \%$ less accurate than valid trials, but there was no effect of object $(F<1)$. A preliminary analysis of RTs showed neither main effects nor interactions with any other factors of display orientation, cue location, target location, or target rotation; therefore, all the data were collapsed across these variables.

An ANOVA conducted on cue validity (invalid vs. valid) revealed a significant effect $[F(1,17)=97.18, p<$ $.01]$, with valid RTs $(M=584 \mathrm{msec})$ being shorter than the invalid RTs $(M=696 \mathrm{msec})$, resulting in a $112-\mathrm{msec}$ validity effect (Figure 4). Responses were much faster to targets presented in a cued location than to those presented in the noncued location, demonstrating the contribution of space-based representation to attentional guidance. Furthermore, an additional ANOVA conducted on object type (SO vs. DO) for invalidly cued targets, failed to reveal a significant difference $(F=1)$ between SO trials $(M=$ $693 \mathrm{msec})$ and DO trials $(M=699 \mathrm{msec})$.

The observed results indicate that object-based representations do not contribute to attentional guidance 


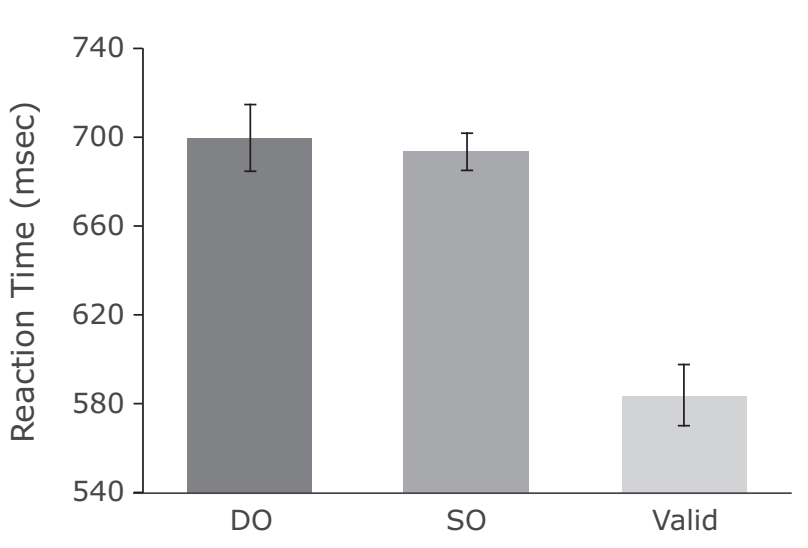

Figure 4. Median correct reaction times in Experiment 2. DO, different object; SO, same object.

when target location is known in advance. Moreover, it is important to note that we replicated our initial finding of Experiment 1 (SOA 400): No object-based effect was present when subjects knew the location of the target with $100 \%$ certainty.

The present experiment, once again, demonstrated that under conditions of $100 \%$ certainty, no object-based effect is observed, suggesting that uncertainty, and not attentional shifting, gives rise to object-based effects. Certainty effectively eliminates the need for the use of object-based representations in attentional guidance and, therefore, eliminates object-based effects. Once subjects have prioritized a spatial location, the object in which the target appears becomes irrelevant. In addition, we demonstrated a large space-based effect, suggesting, not surprisingly, that subjects use space-based representations to guide attention.

\section{EXPERIMENT 3}

It could be argued that the 300-msec SOA employed in Experiment 2 provided ample time for subjects to break fixation and to move eye position to the location of the upcoming target. In order to control for this possibility, an additional experiment was conducted in which eye position was monitored. The goal was to demonstrate that the results of Experiment 2 are replicable when a subject's eye gaze is indeed fixated. The eye movement control ensures that the absence of object-based effects in the previous experiment was not driven by the simple fact that when eye movements were not monitored, the subject simply moved his or her eyes to the cued location. If subjects are able to maintain fixation and still show no sign of an object-based effect, this will provide further support for the attentional prioritization account and will suggest that a priority map is created and attention is allocated in advance only to the target location.

\section{Method}

Subjects. Fourteen subjects (6 male) from George Washington University participated in return for monetary compensation of $\$ 7$. All reported normal or corrected-to-normal visual acuity and were naive as to the purpose of the experiment.
Apparatus and Stimuli. The task and stimuli were exactly the same as those in Experiment 2. This experiment, however, utilized an I-SCAN ETL-500 head-mounted eyetracker, allowing for the tracking of subjects' eye movements.

Design and Procedure. The design and procedure were the same as those in Experiment 2, with the addition of an experimenter response after the subject's response, to indicate whether or not an eye movement was made. The experimenter monitored eye movements on the second computer, and trials on which the eyes deviated from fixation were marked as incorrect.

\section{Results and Discussion}

Only correct responses and trials on which fixation was maintained were included in the analysis. Trials on which the subjects' fixations deviated more than $0.5^{\circ}$ from the central fixation cross were removed ( $6.8 \%$ of the trials). RTs longer than 1,500 msec and shorter than $200 \mathrm{msec}$ were removed according to the trimming procedure, resulting in fewer than $2 \%$ of the trials being discarded. A oneway repeated measures ANOVA on error rates revealed no significant effect of either validity or object $(F<2)$. An ANOVA conducted on RTs with cue validity as a factor revealed a significant cuing effect $[F(1,13)=37.420, p<$ $.01]$ : Valid trials $(M=580 \mathrm{msec})$ were significantly faster than invalid trials $(M=645 \mathrm{msec}$ ) (Figure 5). It should be noted that the validity effect in this experiment (difference of $65 \mathrm{msec}$ ) was smaller than that in the previous experiment (112 msec), suggesting that by maintaining fixation, subjects did not make anticipatory eye movements toward the cued locations. This, in turn, reduced the overall RT difference between the validly and invalidly cued trials.

An additional ANOVA conducted with object type as a factor revealed no effect of object $(F<1)$, corroborating the results obtained in Experiment 2, in which eye movements were not monitored directly. This control experiment effectively ruled out the possibility that the absence of object-based effects was attributable to eye movements, since subjects did not move their eyes either to the validly cued or to the invalidly cued target locations. Moreover, by using the eyetracker, we ruled out the possibility that the absence of object-based effects could have been attributed to an initiation of an eye movement executed to the certain target location before the target onset.

\section{EXPERIMENT 4}

Several conclusions regarding the effect of attentional certainty and attentional shifting on the object-based attentional modulation can be made at this juncture. Shifting of attention is not the necessary component in eliciting object-based effects; rather, what predicts whether object representations guide attentional selection is uncertainty about the upcoming spatial location of the target. Moreover, it was observed that the application of the rules of attentional certainty and the creation of the priority map takes anywhere from 200 to $400 \mathrm{msec}$. So far, the results from all three preceding experiments provide strong evidence for the hypothesis that attentional certainty, and not attentional shifting, elicits object-based effects. However, it should be noted that all three experiments contained 


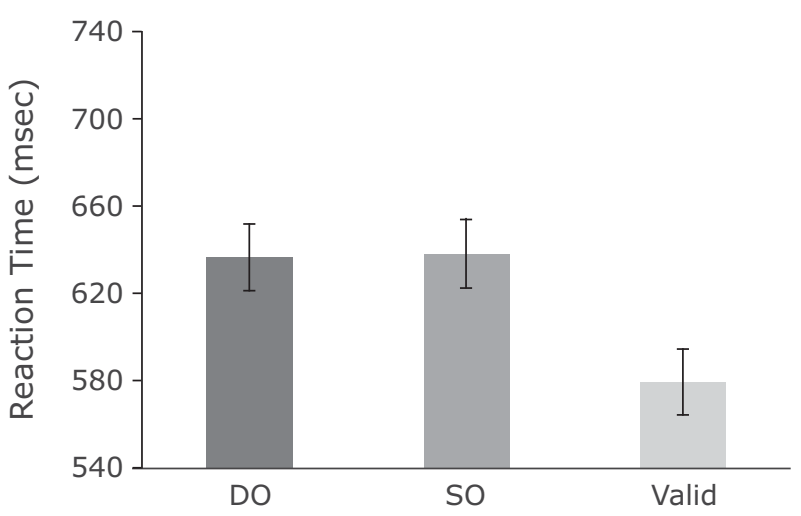

Figure 5. Median correct reaction times in Experiment 3. DO, different object; SO, same object.

shifting and certainty, as in Lamy and Egeth (2002) and Shomstein and Yantis (2002), and it could be argued that in order to successfully dissociate these two mechanisms, either shifting or certainty has to be eliminated.

Therefore, in Experiment 4, attentional shifting was preserved, whereas the knowledge of the spatial location of the upcoming target was eliminated. The prediction was as follows: If attentional certainty is responsible for erasing the contribution of object-based representations to attentional guidance even in the presence of attentional shifts, then, in the absence of attentional certainty, objectbased effects should be recovered.

\section{Method}

Subjects. Seventeen undergraduate students (3 male) from George Washington University participated for extra credit. All reported normal or corrected-to-normal visual acuity.

Apparatus and Stimuli. The apparatus and stimuli were the same as those used as in Experiment 2.

Design and Procedure. The design was the same as that in Experiment 2 . Valid trials were exactly the same and made up $60 \%$ of the total trials. The only difference between Experiment 2 and the present experiment was that the invalid cue no longer predicted the upcoming target location (i.e., half of the targets were presented in the clockwise location, and half in the counterclockwise location), resulting in $20 \%$ each for DO and SO trials. The target had an equal chance of being presented one location clockwise or one location counterclockwise from the cue, and therefore, attention could not be focused on either location until the target appeared.

\section{Results and Discussion}

Only correct responses were included in the analysis. RTs longer than 1,500 msec and shorter than $200 \mathrm{msec}$ were removed, resulting in fewer than $4 \%$ of the trials being discarded. An ANOVA on error rates revealed a significant effect of validity $[F(1,16)=25.482, p<.01]$, such that invalid trials were $3.7 \%$ less accurate than valid trials, but there was no effect of object $(F<1)$. A preliminary RT analysis indicated neither significant main effects nor interactions of display orientation, cue location, target location, target rotation, or target letter; therefore, the data were collapsed over these variables.

An ANOVA was conducted on cue validity (invalid vs. valid), revealing a significant effect $[F(1,16)=124.082$, $p<.01]$ with valid RTs $(M=569 \mathrm{msec})$ being $140 \mathrm{msec}$ shorter than invalid RTs $(M=709 \mathrm{msec})$. Responses were much faster to a cued location than to a noncued location, indicating the contribution of spatial representations to attentional guidance (Figure 6). A one-way ANOVA was conducted on object type (SO vs. DO), which revealed a significant effect $[F(1,16)=17.877, p<.01]$, resulting in a 23-msec object-based effect with SO trials $(M=$ $699 \mathrm{msec})$ yielding shorter RTs than did DO trials $(M=$ $722 \mathrm{msec}$ ).

The aim of this experiment was to examine whether attentional certainty was responsible for the absence of object-based attentional effects in Experiments 1-3. Thus, attentional certainty was removed from this experiment, such that an invalid cue (i.e., the red cue) no longer had any predictive value. If, in fact, the certainty of the target location, and not attentional shifting, gives rise to objectbased attentional effects, reintroducing uncertainty to the paradigm should bring about object-based effects. The results of this experiment demonstrated that when the target location was not known in advance, a strong object-based effect emerged. When the target location was certain, as in the previous experiments, locations were selected before the target appeared. The object-based representation was therefore unnecessary, since subjects depended on spatial representations to guide attentional selection. When target location was uncertain, subjects could not prioritize any particular spatial location prior to target onset and were, thereby, forced to rely, once again, on object-based representations for attentional guidance.

\section{GENERAL DISCUSSION}

In the present study, we set out to investigate the factors influencing attentional guidance by examining the contribution of two alternative mechanisms to object-based selection. In particular, the two examined hypotheses about the use of object-based guidance of attentional selection are the attentional prioritization hypothesis (Müller \& Kleinschmidt, 2003; Shomstein \& Behrmann, 2008; Shomstein \& Yantis, 2002, 2004) and the attentional-

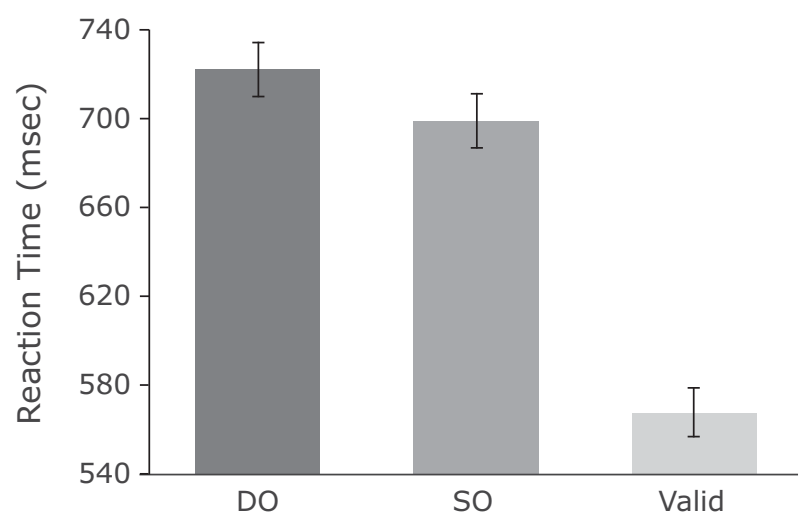

Figure 6. Median correct reaction times from Experiment 4. DO, different object; SO, same object. 
shifting hypothesis (Brown \& Denney, 2007; Lamy \& Egeth, 2002; Watson \& Kramer, 1999). The attentional prioritization hypothesis, in its simplest form, suggests that object-based effects can be attributed to an attentional prioritization mechanism, such that in the absence of an alternative strategy (driven by contingencies, certainty, grouping, etc.), object representations guide attentional selection, and, thus, object-based effects are observed. However, if an alternative strategy presents itself (e.g., the participant knows the target will always appear in a certain location, relative to the cue), object representations are abandoned, and object-based effects are not observed. According to the attentional prioritization hypothesis, when target location is certain, object-based effects will be absent, and this result has indeed been observed and replicated in several investigations (Chan \& Hayward, 2008; Chen \& Cave, 2006 [Experiment 1; but see the rest of the experiments]; Richard et al., 2008; Shomstein \& Behrmann, 2008; Shomstein \& Yantis, 2002, 2004). The attentional-shifting hypothesis, on the other hand, suggests that objects guide attentional selection only when shifts of attention are executed. In other words, if the task itself does not necessitate attentional shifting, object-based effects will not be observed, whereas if attentional shifting is required, object-based effects will manifest (Lamy \& Egeth, 2002).

The present experiments tested the contribution of these two attentional mechanisms by creating circumstances in which the two mechanisms made alternative predictions. The results from the four experiments presented here suggest that the object-based effect is not simply a by-product of attentional shifting. Although attentional shifts are a part of attentional orienting, they do not drive objectbased effects on their own. Rather, the determining factor in whether object representations are used to guide attentional orienting is the presence of an alternative strategythat is, in this particular case, attentional certainty (i.e., knowing the position of the upcoming target in advance). In effect, certainty allows an attentional priority to be assigned to the spatial target location. When attention is focused (i.e., spatial location is prioritized), it is effectively narrowed beyond the object frame of reference, rendering objects unnecessary to the visual search. This interpretation is consistent with Goldsmith and Yeari's (2003) suggestion that the contribution of objects to attentional guidance is related to the type of attention, focused or spread, that is induced by different types of attentional cues (i.e., endogenous or exogenous). Nonpredictive cues result in object-based effects because, following such cues, attention is spread across the display (i.e., diffuse focus of attention), so that when the target appears, objects influence attention. Partially valid cues require attention to be focused on a particular spatial location occupied by the cue, effectively ignoring objects, which eliminates objectbased effects. This diffuse versus narrow attentional focus distinction is consistent with attentional prioritization, such that certainty regarding the position of the upcoming target allows attention to be narrowly focused, thus overriding the influence of objects. Similar results demonstrat- ing that object-based effects are eliminated with certainty have been reported elsewhere in the literature (Goldsmith \& Yeari, 2003; Lavie \& Driver, 1996).

A brief summary of the observed findings clearly provides strong support for the attentional prioritization hypothesis. The results of Experiment 1 demonstrate that when subjects have to shift attention in preparation for a target at a known location, no object-based effects are observed. At a short SOA, however, an object-based effect was observed even in the presence of target certainty. We suggest that even when the target location is known in advance, provided there is not enough time to apply the certainty rule, attention cannot prioritize any specific target location and, therefore, object representations will guide attentional selection (see Shomstein \& Yantis, 2004, for a similar result).

It should be noted that although, initially, it might seem that $200 \mathrm{msec}$ is plenty of time to derive and apply any given certainty rule, the wealth of eye movement literature provides strong evidence that applying a rule relative to a varying sensory event (e.g., a cue that can appear in any one of four locations) is rather time consuming. For example, antisaccades (in which attention is first captured by a cue and then must be redirected away from it) take about $350 \mathrm{msec}$, about $100 \mathrm{msec}$ longer than prosaccades (Butler, Zacks, \& Henderson, 1999; Fischer, Biscaldi, \& Gezeck, 1997; Meriam, Genovese, \& Colby, 2003). We draw a comparison between this time difference and our own certainty rule process, because just as antisaccades require that subjects take information from the cue (e.g., "if the cue appears at location A, direct your attention to location B"), our certainty condition requires subjects to first encode a sensory cue that appears in one of four locations and then to redirect attention to a location in space that is consistent with the imposed certainty rule. This is very similar to the way that invalid trials were designed in the present paradigm; that is, a red cue indicates that attention must be directed away from that location. Further evidence that additional time is required in order to apply a certainty rule comes from van Zoest, Donk, and Theeuwes (2004), who found that goal-driven control influences visual selection at longer SOAs. In other words, being given more time to process cues and rules before the target appears allows those rules to affect visual search patterns more effectively. Therefore, if the certainty rule had already been processed in the 200-msec SOA condition, objects would not have influenced attentional selection (as seen for 400 and $600 \mathrm{msec}$ ). The intent of our inclusion of the RT difference between saccades and antisaccades is not to suggest an exact RT value for this application but, rather, to illustrate the relative increase in timing to accommodate the extra time necessary to apply the certainty rule. With the short time frame of the SOA 200 condition, the priority map is not established before the target appears, thus resulting in an object-based effect (or the inability to filter out the unnecessary object representations).

The results of Experiment 1 were then replicated in Experiment 2, lending further support for the role of attentional certainty, and not attentional shifting, in atten- 
tional guidance. In addition, Experiment 3 eliminated a possible criticism, that the results of pervious experiments could have been attributable to subjects' breaking fixation and moving their eyes to the location of the upcoming target. Finally, in Experiment 4, attentional shifting was kept the same as in Experiments 1, 2, and 3 but this time, attentional certainty regarding the spatial location of the upcoming target was removed. As a result of this manipulation, invalidly cued targets could appear one location clockwise or counterclockwise. Due to the uncertain nature of the target location, subjects could not prioritize any spatial location in advance of the target, ultimately being left to rely on object representations for attentional guidance.

A potential alternative interpretation of our data could possibly be put forward. One might argue that the reason object-based effects were absent when subjects had 100\% certainty as to the location of the invalidly cued target (Experiment 2) and were present when subjects were uncertain about the upcoming invalidly cued target (Experiment 4) is because, in the certainty experiment, subjects could initiate an attentional shift (or initiate an eye movement) to the target location before the onset of the target (or right after the cue). In the uncertainty experiment (Experiment 4), such anticipatory shifts of attention (or of eye position) were, of course, impossible, because subjects were uninformed as to the location of the upcoming target. Although this alternative interpretation seems reasonable at a first glance, a more careful examination of the results presents strong evidence against such interpretation.

We first address the alternative interpretation involving possible eye movements that are initiated following the onset of the cue in preparation to the target onset. Of course, this explanation is plausible, since an SOA of $400 \mathrm{msec}$ allows plenty of time for planning and executing a saccade. In order to rule out this alternative explanation, we conducted Experiment 3, in which eye position was monitored while subjects performed the same certainty experiment as in Experiment 2. In the absence of any eye movements, we observed that certainty led to an absence of object-based effects. This control experiment effectively ruled the possibility that the absence of objectbased effects was attributable to eye movements, thus providing support for the attentional-shifting hypothesis, since subjects did not move their eyes to the target location but, rather, assigned highest priority to the target location on the basis of the formed priority map. This map made shifts unnecessary and resulted in the elimination of the object-based effect.

We now turn our attention to the possibility that when the target location was known with certainty (as in Experiment 2), subjects were able to initiate and complete an attentional shift after the onset of the cue and before the onset of the target. Such anticipatory shifts would be impossible to initiate when the target location is unknown in advance (i.e., uncertainty in Experiment 4). Two lines of evidence, drawn from our data, provide strong support against this alternative explanation.

The first piece of evidence is gathered from examining the magnitude of the attentional-cuing effect when the location of the invalidly cued target was certain (Experiments 2 and 3) and when it was uncertain (Experiment 4). The logic is as follows: If attention was shifted to the location of the invalid target before the target appeared on the screen, we would observe a decrease in the magnitude of the cuing effect (as compared with Experiment 4, in which invalid target location was uncertain and attentional shift could be initiated only after the target onset). Our data clearly do not show this pattern and, therefore, support our original hypothesis. First, there was a significant spatialcuing effect in both experiments, suggesting that attentional shifts, in both experiments (Experiments 2 and 4), took place only after the targets and distractors were presented, rather than between the cue and the target onsets. Second, the magnitude of this cuing effect was absolutely identical in Experiments 2, 3, and 4. This was verified by conducting an ANOVA with validity as a within-subjects factor and experiment as a between-subjects factor. The ANOVA yielded no significant interaction between validity and certainty, thus indicating that the magnitude of the validity effect was similar in both experiments $(F<1)$.

It could be argued, however, that it is not surprising that the cuing effect turned out not to be significant in the above-mentioned ANOVA, since it could be that there was simply insufficient power to detect a $28-\mathrm{msec}$ effect. To ensure that the null effect was not due to lack of power, we conducted a secondary ANOVA on objectbased effects. Since object-based effects were absent in Experiments 2 and 3 and were present in Experiment 4, finding a significant interaction between experiment and object would demonstrate sufficient statistical power. An ANOVA was conducted with object as a within-subjects factor and experiment as a between-subjects factor, first for Experiments 2 and 4, yielding a significant interaction $[F(1,33)=4.833, p<.05]$. The same ANOVA was conducted on Experiments 3 and 4, also yielding a significant interaction $[F(1,31)=10.147, p<.01]$. This is important because it provides further evidence that (1) the results of the previously mentioned ANOVA for validity were not due to a lack of statistical power, (2) the presence of the object-based effect was modulated by the experiment and, therefore, by the inclusion of target location certainty (since all other aspects of the experiment were identical), and (3) the results obtained were similar across the experiments in which eye movement position was monitored and that in which subjects were simply asked to fixate, providing further evidence that the observed findings cannot be attributable to anticipatory attentional shifts toward the target location.

The second piece of evidence that can be used to argue against the aforementioned anticipatory attentionalshifting hypothesis is drawn from the fact that the overall RTs in invalid conditions when target location was known in advance (Experiment 2; see Figure 4) were not shorter than the invalid condition RTs for the experiment in which target location was unknown (Experiment 4; see Figure 6). The logic is as follows: If $400 \mathrm{msec}$ is enough to fully execute a shift to the location of the upcoming target when that location is known in advance, RTs to identify the target letter should be shorter than when the shift is initiated 
only after the target appears (when target location is not known in advance). However, this pattern of results was not observed. In fact, RTs for the invalidly cued targets in both experiments ( 2 and 3 ) are comparable, and the most convincing piece of evidence comes from the fact that not only are the RTs from invalid conditions comparable, but both valid conditions show very similar RTs (584 msec in Experiment 2 and $569 \mathrm{msec}$ in Experiment 4). The latter RT comparison demonstrates that the two samples of subjects were roughly equivalent in terms of the efficiency of their overall manual responses. These statements were supported by the results of an auxiliary ANOVA in which overall RTs for invalid and valid conditions were compared between the two experiments (interaction, $F<1$; but with significant main effects in both Experiment 2 and Experiment 4). It is important to note that the only change from Experiment 2 to Experiment 4 was the predictive value of the red cue. In Experiment 2, the red cue indicated that a target would appear one location clockwise from the cue, and in Experiment 4, it indicated that any one of the two noncued locations could contain a target. Therefore, in both cases, subjects had to determine whether they should focus on the cued location (blue) or direct attention away (red). The same amount of cue processing and search was necessary in both experiments, which would further explain and support the similar RTs. Therefore, given that the alternative interpretation (based on eye movements or on anticipatory attentional shifts) is not supported by the observed results, we conclude that attentional prioritization, and not attentional shifting, gives rise to object-based effects. $^{2}$

Recently, however, the attentional prioritization hypothesis and the role of attentional certainty, in particular, have come under scrutiny in several investigations. Studies by Chen and Cave (2006, 2008), for example, have suggested that the reason that object-based effects do not manifest themselves under conditions of $100 \%$ attentional certainty is not due to the successful narrowing of attention on the target location, as was initially argued by Shomstein and Yantis (2002), but, rather, is due to the type of representational display that was employed. In particular, Chen and Cave $(2006,2008)$ suggested that the three rectangles arranged into a crosslike configuration, used by Shomstein and Yantis (2002), were interpreted not as three independent objects, but rather as a single multipart object (i.e., a cross). To demonstrate that such alternative interpretation of the display could result in the absence of object-based effects, the authors first replicated the original finding observed by Shomstein and Yantis (2002) and then went on to ensure that subjects did, in fact, perceive the three rectangles as three independent objects by decomposing the three-rectangle display into the component pieces: On some trials, subjects were presented with the full display (three rectangles), with two smaller rectangles alone, or with one long rectangle alone. It was observed that by manipulating how much of the display was shown, even when target location was known in advance with $100 \%$ certainty, object-based effects were present. Chen and Cave $(2006,2008)$ concluded that object representations guide attentional selection even when the location of the upcoming target is known in advance, if the display is interpreted as consisting of different objects.

Several important factors need to be considered when interpreting the findings observed by Chen and Cave (2006, 2008), and although the observed findings seem inconsistent with attentional prioritization at first glance, they can nevertheless be integrated with the attentional prioritization hypothesis rather seamlessly. Attentional prioritization argues that target location certainty eliminates object-based effects, because it allows for attention to narrowly focus and prioritize a spatial location, which effectively filters out irrelevant object representations. In Chen and Cave's (2006) manipulation, some trials contained the full cross (three objects), whereas some trials contained only pieces of the display (either two short rectangles or one long rectangle). They suggested that the trials with a partial display affected attentional selection on trials with a complete display, so that when the full cross appeared, subjects interpreted the three-rectangle display as consisting of three different objects (rather than as interpreting it as a single crosslike display). However, it should be noted that even though, in that paradigm, subjects were $100 \%$ certain about the location of the upcoming target, a different type of uncertainty was introduced into the display - that of configural uncertainty. In other words, from trial to trial, subjects were presented with three different configural displays. Therefore, even when the target's location was known in advance, the lack of certainty about the completeness of the display was enough to alter attentional allocation. Namely, this uncertainty placed an emphasis back onto the objects and, therefore, resulted in an inability to successfully filter out object representations, thus leading to an object-based effect.

In their 2008 experiments, Chen and Cave again found object-based effects with positional certainty. However, attention was split between two targets, so that even when the target locations were certain, attention was focused on multiple locations at once. Prioritizing multiple locations is what occurs under conditions of target location uncertainty. In addition, Chen and Cave's (2008) results can be reconciled with the attentional prioritization hypothesis by framing their findings within Goldsmith and Yeari's (2003) attentional focus hypothesis. Goldsmith and Yeari suggested that when attention is spread across the display (i.e., diffuse attentional focus), objects contribute to attentional guidance, whereas focused attention (i.e., narrow attentional focus) allows the objects to become irrelevant and, therefore, they are successfully filtered out and do not guide attention. Perhaps, as Goldsmith and Yeari suggested, the changing of the display configuration on a trial-by-trial basis led to a diffuse state of attention, which would lend itself to object-based effects. Further investigation is necessary to determine what accounts for the discrepancy between our results and those of Chen and Cave (2006, 2008), whether it is certainty (configural or positional) or whether the cue creates a diffuse spread of attention that is not conducive to attentional prioritization. Regardless of the outcome of future investigations, however, it should be noted that the results of Shomstein and Yantis (2002) were successfully corroborated in the experiments described in this 
article, experiments that employed the robust and widely adopted two-rectangle paradigm (Egly et al., 1994). Few would argue that the two-rectangle display is interpreted as anything other than two independent rectangles. As such, the present results showing that object-based effects are not present when target location is known in advance with $100 \%$ certainty serve as strong evidence for the support of the attentional prioritization hypothesis.

Another recent study, conducted by Richard et al. (2008), argued that although object-based effects are indeed observed under conditions of $100 \%$ certainty, this outcome is due to the perception that the targets are a part of (i.e., belong) to the object shape. The authors contended that Shomstein and Yantis (2002) did not observe objectbased effects in their $100 \%$ target certainty paradigm because the target letters (Ts and Ls) were interpreted as being "placed" on top of the object, rather than perceived as being parts of the object (Richard et al., 2008). Although this alternative interpretation is interesting and is supported by several experiments (Richard et al., 2008), it does not explain why Shomstein and Yantis (2002) did observe object-based effects in their original study when the factor being manipulated was the predictive value of the cue (i.e., the target location was no longer certain), and not aspects of the physical display. In addition, Richard et al.'s account does not explain why, in our present experiment, with the same type of targets (letters Ts and Ls), we did not observe object-based effects when target location was known in advance and did observe object-based effects when target location was determined randomly. Again, further experiments will have to be conducted in order to reconcile these apparently opposing interpretations.

There are multiple factors involved in object-based attention. Our results suggest that the effect is not simply a result of attentional shifting; there is another factor driving the implementation of shifts. Certainty of target location allows the participant to prioritize the target location, and shifting occurs with the same efficiency within or between the objects. When, on the other hand, target location is uncertain (as in Experiment 3), an object-based effect is present, because objects are a necessary component of the search for the target. Without advance knowledge, attention must use all available representations in its search (space and object based), and therefore, which object the target appears within plays a role in orienting. However, when target location is $100 \%$ certain (as in Experiments 1 and 2), that knowledge can be applied, and attention is allowed to focus narrowly on the target location while filtering out the objects. Under those conditions, objects are not a necessary resource for the target search, resulting in no object-based effect.

In summary, on the basis of the experiments reported here, as well as others that have investigated the mechanism of object-based attentional selection, we suggest that object-based effects are most parsimoniously explained by the attentional prioritization mechanism of attentional guidance. Object representations will guide attentional selection only in the absence of an alternative strategy - that is, ceteris paribus. However, once an alternative strategy presents itself, be it based on location certainty of the up- coming target, perceptual organization of the display, or something else, object representations are abandoned in lieu of a more efficient attentional guidance strategy.

\section{AUTHOR NOTE}

We thank Ashleigh Richard, Dominique Lamy, and Kyle Cave for helpful and constructive comments on earlier versions of the manuscript. We also thank John Philbeck for sharing the eyetracking equipment and Dan Gajewski for assistance during the eye movement data acquisition phase. Correspondence should be addressed to L. Drummond, Department of Psychology, George Washington University, Washington, DC 20052 (e-mail: drummond@gwmail.gwu.edu).

\section{REFERENCES}

Avrahami, J. (1999). Objects of attention, objects of perception. Perception \& Psychophysics, 61, 1604-1612.

Behrmann, M., Zemel, R. S., \& Mozer, M. C. (1998). Object-based attention and occlusion: Evidence from normal participants and a computational model. Journal of Experimental Psychology: Human Perception \& Performance, 24, 1011-1036. doi:10.1037/0096-1523 .24.4.1011

Brawn, P. T., \& SNOWden, R. J. (2000). Attention to overlapping objects: Detection and discrimination of luminance changes. Journal of Experimental Psychology: Human Perception \& Performance, 26, 342-358.

Brown, J. M., \& Denney, H. I. (2007). Shifting attention into and out of objects: Evaluating the processes underlying the object advantage. Perception \& Psychophysics, 69, 606-618. doi:10.1037/0096 $-1523.26 .1 .342$

Butler, K., Zacks, R., \& Henderson, J. (1999). Suppression of reflexive saccades in younger and older adults: Age comparisons on an antisaccade task. Memory \& Cognition, 27, 584-591.

Cave, K. R., \& Wolfe, J. M. (1990). Modeling the role of parallel processing in visual search. Cognitive Psychology, 22, 225-271. doi:10.1016/0010-0285(90)90017-X

Cepeda, N. J., \& Kramer, A. F. (1999). Strategic effects on objectbased attentional selection. Acta Psychologia, 103, 1-19. doi:10.1016/ S0001-6918(99)00021-9

Chan, L. K. H., \& Hayward, W. G. (2008, November). Object-based attention is "turned off" by top-down control. Paper presented at the 16th Annual Workshop on Object Perception, Attention, \& Memory, Chicago.

Chen, Z., \& Cave, K. R. (2006). Reinstating object-based attention under positional certainty: The importance of subjective parsing. Perception \& Psychophysics, 68, 992-1003.

Chen, Z., \& CAVE, K. R. (2008). Object-based attention with endogenous cuing and positional certainty. Perception \& Psychophysics, 70, 1435-1443.

Corbetta, M., Miezin, F. M., Dobmeyer, S., Shulman, G. L., \& PeTERSEN, S. E. (1990). Attentional modulation of neural processing of shape, color, and velocity in humans. Science, 248, 1556-1559. doi: $10.1126 /$ science. 2360050

Duncan, J. (1984). Selective attention and the organization of visual information. Journal of Experimental Psychology: General, 113, 501517. doi:10.1037/0096-3445.113.4.501

Egly, R., Driver, J., \& Rafal, R. D. (1994). Shifting visual attention between objects and locations: Evidence from normal and parietal lesion subjects. Journal of Experimental Psychology: General, 123, 161-177. doi:10.1037/0096-3445.123.2.161

Eriksen, B. A., \& Eriksen, C. W. (1974). Effects of noise letters upon the identification of a target letter in a nonsearch task. Perception \& Psychophysics, 16, 143-149.

Fischer, B., Biscaldi, M., \& Gezeck, S. (1997). On the development of voluntary and reflexive components in saccade generation. Brain Research, 754, 285-297.

Goldsmith, M. (1998). What's in a location? Comparing object-based and space-based models of feature integration in visual search. Journal of Experimental Psychology: General, 127, 189-219. doi:10 1037/0096-3445.127.2.189

Goldsmith, M., \& Yeari, M. (2003). Modulation of object-based attention by spatial focus under endogenous and exogenous orienting. 
Journal of Experimental Psychology: Human Perception \& Performance, 29, 897-918. doi:10.1037/0096-1523.29.5.897

HARMS, L., \& BundESEN, C. (1983). Color segregation and selective attention in a nonsearch task. Perception \& Psychophysics, 33, 11-19.

KIM, M. S., \& CAVE, K. R. (2001). Perceptual grouping via spatial selection in a focused-attention task. Vision Research, 41, 611-624. doi:10.1016/S0042-6989(00)00285-6

Kramer, A. F., \& Jacobson, A. (1991). Perceptual organization and focused attention: The role of objects and proximity in visual processing. Perception \& Psychophysics, 50, 267-284.

LAMY, D., \& EgETH, H. (2002). Object-based selection: The role of attentional shifts. Perception \& Psychophysics, 64, 52-66.

LAVIE, N., \& Driver, J. (1996). On the spatial extent of attention in objectbased visual selection. Perception \& Psychophysics, 58, 1238-1251.

Meriam, E., Genovese, C., \& Colby, C. (2003). Spatial updating in human parietal cortex. Neuron, 39, 361-373.

Moore, C. M., Yantis, S., \& Vaughan, B. (1998). Object-based visual selection: Evidence from perceptual completion. Psychological Science, 9, 104-110. doi:10.1111/1467-9280.00019

Müller, N. G., \& KleinschmidT, A. (2003). Dynamic interaction of object- and space-based attention in retinotopic visual areas. Journal of Neuroscience, 23, 9812-9816.

O'Craven, K. M., Downing, P. E., \& Kanwisher, N. (1999). fMRI evidence for objects as the units of attentional selection. Nature, 401, 584-587. doi:10.1038/44134

Richard, A. M., Lee, H., \& Vecera, S. P. (2008). Attentional spreading in object-based attention. Journal of Experimental Psychology: Human Perception \& Performance, 34, 842-853. doi:10.1037/0096 $-1523.34 .4 .842$

Rock, I., \& GutTman, D. (1981). The effect of inattention on form perception. Journal of Experimental Psychology: Human Perception \& Performance, 7, 275-278. doi:10.1037/0096-1523.7.2.275

Roelfsema, P. R., Lamme, V. A. F., \& Spekreijse, H. (1998). Objectbased attention in the primary visual cortex of the macaque monkey. Nature, 395, 376-381. doi:10.1038/26475

Shomstein, S., \& Behrmann, M. (2006). Cortical systems mediating visual attention to both objects and spatial locations. Proceedings of the National Academy of Sciences, 103, 11387-11392. doi:10.1073/ pnas. 0601813103
Shomstein, S., \& Behrmann, M. (2008). Object-based attention: Strength of object representation and attentional guidance. Perception \& Psychophysics, 70, 132-144. doi:10.3758/PP.70.1.132

Shomstein, S., \& Yantis, S. (2002). Object-based attention: Sensory modulation or priority setting? Perception \& Psychophysics, 64, 41-51.

Shomstein, S., \& Yantis, S. (2004). Configural and contextual prioritization in object-based attention. Psychonomic Bulletin \& Review, 11, 247-253.

VAn Zoest, W., Donk, M., \& Theeuwes, J. (2004). The role of stimulusdriven and goal-driven control in saccadic visual selection. Journal of Experimental Psychology: Human Perception \& Performance, 30, 746-759.

Watson, S. E., \& Kramer, A. F. (1999). Object-based visual selective attention and perceptual organization. Perception \& Psychophysics, 61, 31-49.

WolfE, J. M. (1994). Guided Search 2.0: A revised model of visual search. Psychonomic Bulletin \& Review, 1, 202-238.

Zemel, R. S., Behrmann, M., Mozer, M., \& Bavelier, D. (2002). Experience-dependent perceptual grouping and object-based attention. Journal of Experimental Psychology: Human Perception \& Performance, 28, 202-217. doi:10.1037/0096-1523.28.1.202

\section{NOTES}

1. Although both alternative hypotheses challenge the sensory enhancement account, the debate is still ongoing, particularly since there are several inconsistent findings that need to be integrated. For example, both attentional prioritization and attentional shifting seem to be inconsistent with some findings reported in the literature (Chen \& Cave, 2006; Harms \& Bundesen, 1983; Kim \& Cave, 2001; Kramer \& Jacobson, 1991). Further investigations are warranted in order to resolve the remaining inconsistencies.

2. It should be noted that it is not argued here that anticipatory shifts cannot occur in a 400-msec time window between the cue and the target. Although such shifts are possible, it is demonstrated with empirical evidence that such shifts do not occur.

(Manuscript received April 1, 2009; revision accepted for publication April 24, 2010.) 